\title{
Supernova Neutrinos in Future Liquid-Scintillator Detectors
}

\author{
Yu-Feng $\mathbf{L i}^{* *}$ \\ Institute of High Energy Physics, Chinese Academy of Sciences, Beijing 100049, China \\ E-mail: liyufengeihep.ac.cn
}

\begin{abstract}
A high-statistics measurement of the neutrinos from a galactic core-collapse supernova ( $\mathrm{SN})$ is extremely important for understanding the explosion mechanism, and studying the intrinsic properties of neutrinos. Large liquid-scintillator detectors can be implemented to observe Galactic SN neutrinos in various reaction channels, including the inverse beta decay reaction on free protons, the elastic neutrino-proton scattering and neutrino-electron scattering, as well as the chargedcurrent and neutral-current interactions on the carbon nuclei. In this talk, we shall illustrate that a global analysis of all these channels is very important to test the average-energy hierarchy of SN neutrinos and how the total energy is partitioned among neutrino flavors. The precision levels for the total and averaged energies of three different neutrino flavors will be also discussed. In addition, using the event time distribution of the inverse beta decay channel, the upper bound on the absolute neutrino masses is found to be $m_{v}<(0.83 \pm 0.24) \mathrm{eV}$ at the $95 \%$ confidence level (C.L.) for a typical galactic supernova, assuming a nearly-degenerate neutrino mass spectrum and a normal mass ordering. Finally, we stress that the liquid-scintillator, water-cherenkov and liquid-argon detectors are complementary in the future SN neutrino observation.
\end{abstract}

Neutrino Oscillation Workshop

4 - 11 September, 2016

Otranto (Lecce, Italy)

\footnotetext{
* Speaker.

$\dagger$ This work was supported in part by the National Natural Science Foundation of China under Grant Nos. 11135009 and 11305193, by the Strategic Priority Research Program of the Chinese Academy of Sciences under Grant No. XDA10010100, and by the CAS Center for Excellence in Particle Physics.
} 


\section{Introduction}

A massive star is expected to experience a core collapse under its own gravity, and then a violent explosion [U]. In the paradigm of neutrino-driven explosion, it is neutrinos that restore most of the gravitational binding energy released in the core collapse and deposit part of it to the surroundings, reviving the halted shock wave and leading to a successful explosion []]. Therefore, the detection of neutrino signals and reconstruction of neutrino energy spectra are of crucial importance to verify the neutrino-driven paradigm, and finally reveal the true mechanism for corecollapse supernova (SN) explosions [[]].

The neutrino burst from SN 1987A in the Large Magellanic Cloud was clearly recorded in the Kamiokande-II [3], Irvine-Michigan-Brookhaven (IMB) [团], and Baksan [1] experiments. Although only twenty-four neutrino events in total were observed, we have acquired very useful information about the explosion mechanism of core-collapse $\mathrm{SNe}$ and the intrinsic properties of neutrinos themselves [ [6].

The interest in SN neutrino observation has recently been stimulated by the great progress in neutrino oscillation experiments, for which large water-Cherenkov, liquid-scintillator and liquidargon detectors will be on-line in the near future. For a galactic core-collapse SN at a typical distance of $10 \mathrm{kpc}$, the Super-Kamiokande detector is capable of collecting about $10^{4}$ neutrino events mainly in the inverse beta decay (IBD) channel. As for the liquid-scintillator detector of the Jiangmen Underground Neutrino Observatory (JUNO), it will register about 5000 events from IBD, 2000 events from all-flavor elastic neutrino-proton scattering, with more than 300 events from neutrino-electron scattering, and hundreds of events from charged-current and neutral-current interactions on the carbon nuclei. Regarding the liquid-argon detector (i.e., DUNE), the dominant channel is the electron-neutrino charged-current interaction on the argon nuclei. Therefore, different SN neutrino detectors are complementary and the prospective of SN neutrino observation in the future is very promising.

\section{Supernova neutrinos in liquid-scintillator detectors}

Future large liquid-scintillator detectors will have several advantages for the detection of SN neutrinos. First of all, the threshold of visible energy in a liquid-scintillator detector could be rather low, since it is only limited by the intrinsic radioactive backgrounds of the liquid scintillator itself. In this case, the elastic neutrino-proton scattering becomes very important, giving rise to a large number of events in a channel other than IBD. Second, the carbon nuclei in the liquid-scintillator serve as an invaluable target for $\mathrm{SN}$ neutrino detection. In particular, the $\mathrm{SN} v_{e}$ is detectable via the charged-current interaction on ${ }^{12} \mathrm{C}$ in addition to the elastic scattering off electrons and protons. Third, it is possible to distinguish between protons and electrons in a liquid-scintillator detector with the pulse shape discrimination, implying a remarkable reduction of background in each channel. Finally, the charged-current and neutral interaction on ${ }^{13} \mathrm{C}$ might also be promising channels to detect SN neutrinos. Therefore, the liquid-scintillator detectors are able to provide us with more information about neutrino flavors and energy spectra. Taking the $20 \mathrm{kton}$ liquid-scintillator detector of JUNO [ $[$ ] for illustration, the SN neutrino event numbers of six different reaction channels for a core-collapse SN at a typical distance of $10 \mathrm{kpc}$ are summarized in Tab. 1. For the elastic proton 


\begin{tabular}{lcccc}
\hline \multirow{2}{*}{ Channel } & \multirow{2}{*}{ Type } & \multicolumn{3}{c}{ Number of SN Neutrino Events at JUNO } \\
\cline { 3 - 5 } & & No Oscillations & Normal Ordering & Inverted Ordering \\
\hline $\bar{v}_{e}+p \rightarrow e^{+}+n$ & $\mathrm{CC}$ & 4573 & 4775 & 5185 \\
$v+p \rightarrow v+p$ & $\mathrm{ES}$ & 1544 & 1544 & 1544 \\
$v+e \rightarrow v+e$ & $\mathrm{ES}$ & 313 & 315 & 315 \\
$v+{ }^{12} \mathrm{C} \rightarrow v+{ }^{12} \mathrm{C}^{*}$ & $\mathrm{NC}$ & 352 & 352 & 352 \\
$v_{e}+{ }^{12} \mathrm{C} \rightarrow e^{-}+{ }^{12} \mathrm{~N}$ & $\mathrm{CC}$ & 43 & 134 & 106 \\
$\bar{v}_{e}+{ }^{12} \mathrm{C} \rightarrow e^{+}+{ }^{12} \mathrm{~B}$ & $\mathrm{CC}$ & 86 & 98 & 126 \\
\hline
\end{tabular}

Table 1: Numbers of neutrino events at JUNO for a core-collapse SN at a typical distance of $10 \mathrm{kpc}$ [ [D]. The gravitational binding energy of $3 \times 10^{53}$ erg is assumed to be equally distributed in all six neutrino and antineutrino species, and the average neutrino energies are $\left\langle E_{v_{e}}\right\rangle=12 \mathrm{MeV},\left\langle E_{\bar{v}_{e}}\right\rangle=14 \mathrm{MeV}$ and $\left\langle E_{v_{x}}\right\rangle=16 \mathrm{MeV}$. For the elastic proton and electron scattering, a threshold of $0.2 \mathrm{MeV}$ for the recoil energy is chosen.

and electron scattering events, a threshold of $0.2 \mathrm{MeV}$ for the recoil energy is chosen. With these SN neutrino events, one can discuss the physics potential both in particle physics and astrophysics. In the following we shall present the precision measurements of astrophysical parameters [[]] and constraining the absolute neutrino masses [9], respectively.

\subsection{Global analysis of different reaction channels}

Combining all the possible reaction channels, one can make a global analysis of the SN neutrino events. First, the $\bar{v}_{e}$ flavor is detected in the IBD and ${ }^{12} \mathrm{C}-\mathrm{CC}$ processes, which according to Table $\mathbb{W}$ have the event statistics of around 5000 and 100, respectively. Therefore, the parameters of the $\bar{v}_{e}$ flavor are determined predominately by the IBD channel, which is shown in the left panel of Fig. $\mathrm{W}$. The precision of the luminosity and average energy at the $90 \%$ confidence level (C.L.) in the IBD channel are 5.4\% and 1.4\%, respectively. Second, in the middle panel of Fig. I, we show the allowed regions of the luminosity and average energy of $v_{e}$ in the individual fitting of the $e \mathrm{ES}$, ${ }^{12} \mathrm{C}-\mathrm{CC}$ and ${ }^{13} \mathrm{~N}-\mathrm{CC}$ processes. The accuracies of $E_{v_{e}}^{\text {tot }}$ and $\left\langle E_{v_{e}}\right\rangle$ in the global fitting scenario are $24 \%$ and $12 \%$, respectively. Third, in the right panel of Fig. 四, we illustrate the allowed regions of the luminosity and average energy at the $90 \%$ C.L. in the individual fitting of the $e \mathrm{ES}+{ }^{13} \mathrm{~N}-\mathrm{CC}$, $p \mathrm{ES},{ }^{12} \mathrm{C}-\mathrm{NC}$ and ${ }^{13} \mathrm{C}-\mathrm{NC}$ processes. Combining these complementary processes, the precision levels of the luminosity and average energy turn out to be $27 \%$ and $4.6 \%$, respectively. Finally, the global analysis can also be used to test the average-energy hierarchy of SN neutrinos and how the total energy is partitioned among neutrino flavors, which are thoroughly discussed in Ref. []].

\subsection{Constraining the absolute neutrino masses}

Compared to that of massless particles, the flight time of massive neutrinos from the SN to the detector at the Earth will be delayed. Therefore, the absolute neutrino masses can be tested using the time distribution of SN neutrino events. Here we employ the two-dimensional time and energy spectra of SN IBD events. Based on a simple but useful model of SN neutrino fluxes and the maximum likelihood analysis, we have carried out a number of simulations to explore the upper bound on absolute neutrino masses at JUNO in Fig. ㅁ. In assumption of a nearly-degenerate mass 

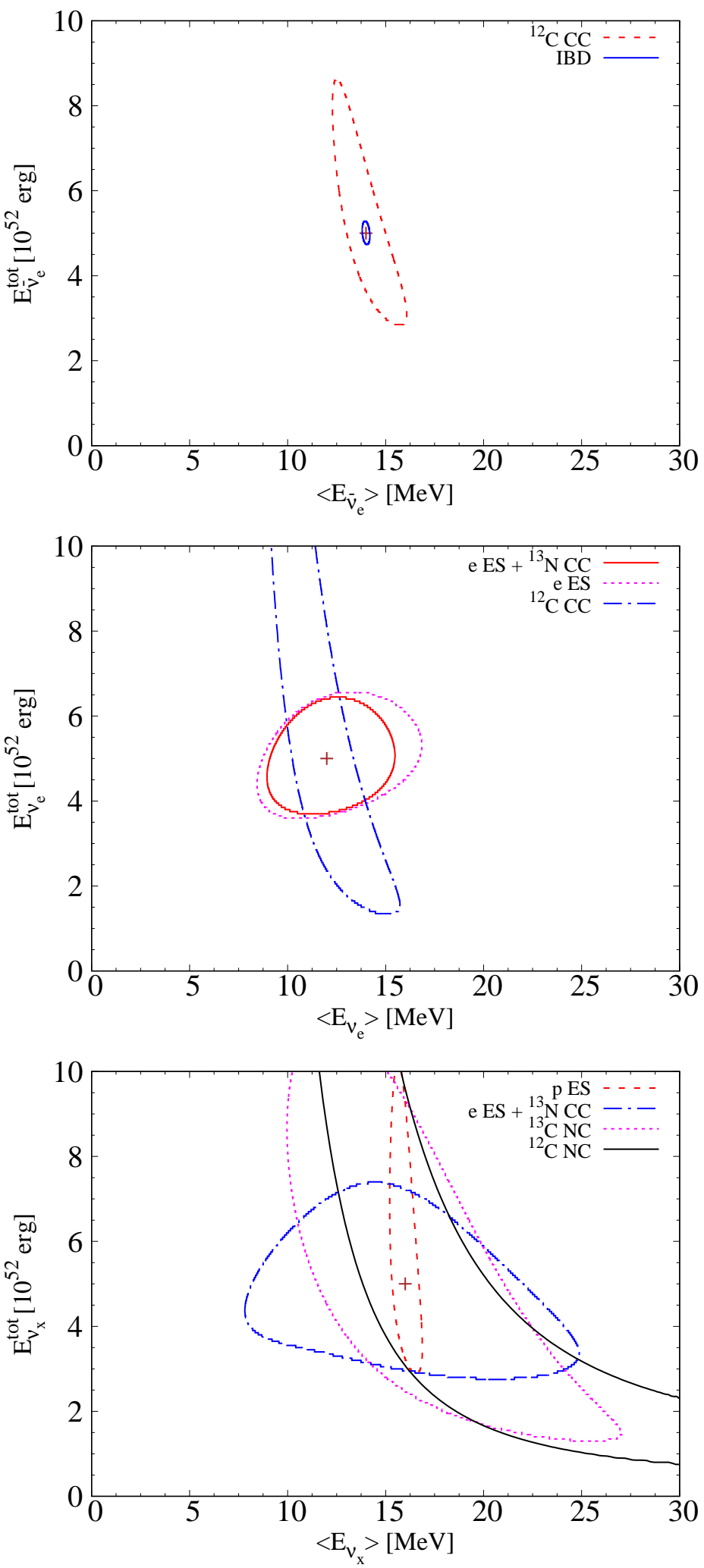

Figure 1: Allowed regions of the luminosities and average energies of $\bar{v}_{e}$ (upper panel), $v_{e}$ (middle panel), $v_{x}$ (lower panel) at the $90 \%$ C.L. in the individual fitting of different reaction channels [ $[8]$. 


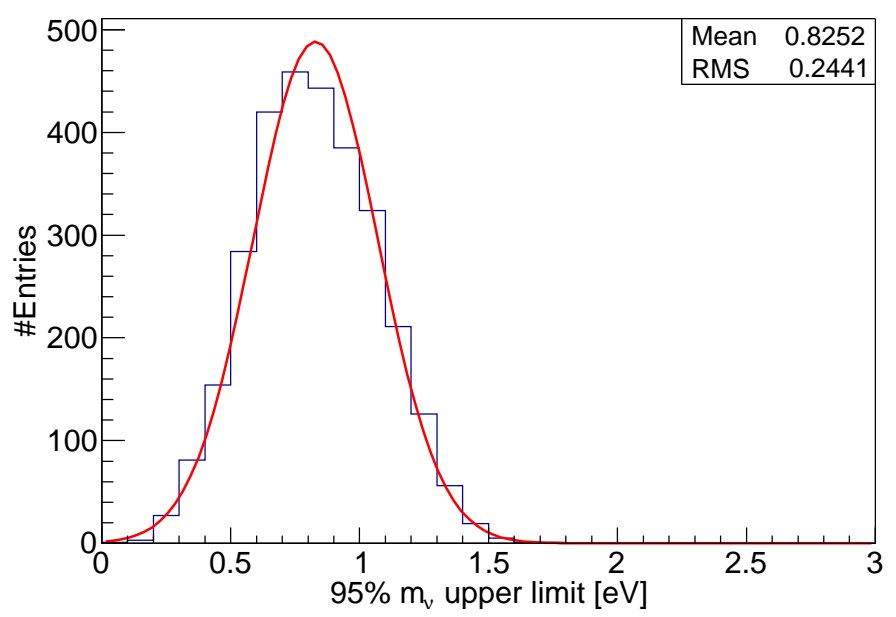

Figure 2: Histogram of the 95\% upper limit on neutrino masses for 3000 simulations at JUNO [Q] A A Gaussian fit to the histogram has been performed and shown together with the mean value and standard deviation.

spectrum and a normal mass ordering, it is found that $m_{v}<(0.83 \pm 0.24) \mathrm{eV}$ at the $95 \%$ C.L. can be reached.

\section{Concluding remarks}

Future large liquid-scintillator detectors will offer us a great opportunity to detect galactic SN neutrinos in a number of different channels, which can be used to reconstruct average neutrino energies and the total gravitational binding energy. In addition, the real-time measurement of SN neutrinos is also precious to probe the absolute neutrino masses using the time-delay effects. All the information is indispensable for us to understand the dynamics of a core-collapse $\mathrm{SN}$ as well as fundamental properties of particle physics.

\section{References}

[1] H. A. Bethe, Rev. Mod. Phys. 62, 801 (1990).

[2] A. Mirizzi et al., Riv. Nuovo Cim. 39, 1 (2016) [arXiv:1508.00785].

[3] K. Hirata et al. [KAMIOKANDE-II Collaboration], Phys. Rev. Lett. 58, 1490 (1987).

[4] R. M. Bionta et al., Phys. Rev. Lett. 58, 1494 (1987).

[5] E. N. Alekseev, L. N. Alekseeva, I. V. Krivosheina and V. I. Volchenko, Phys. Lett. B 205, 209 (1988).

[6] G. G. Raffelt, Stars as Laboratories for Fundamental Physics (Chicago University Press, Chicago, 1996).

[7] F. An et al. [JUNO Collaboration], J. Phys. G 43, no. 3, 030401 (2016) [arXiv:1507.05613].

[8] J. S. Lu, Y. F. Li and S. Zhou, Phys. Rev. D 94, 023006 (2016) [arXiv:1605.07803].

[9] J. S. Lu, J. Cao, Y. F. Li and S. Zhou, JCAP 1505, 044 (2015) [arXiv:1412.7418]. 